\title{
DONNA IMPEGNATA A LIVELLO CIVILE E CULTURALE: DORA D'ISTRIA LA VOCE DI UNA INTELLETTUALE FUORI DAL CORO
}

\author{
Woman Involved on a Civil and Cultural Level: Dora d'Istria \\ the Voice of an Intellectual Outside the Box
}

\author{
Vittoria Bosna \\ vittoria.bosna@uniba.it \\ Università degli Studi di Bari «Aldo Moro». Italia \\ Fecha de recepción: 02/03/2019 \\ Fecha de aceptación: 01/07/2019
}

\begin{abstract}
Elena Ghika, conosciuta con il nome d'arte di Dora d'Istria, donna istruita ed erudita, si inserisce nel dibattito sulla emancipazione civile e culturale femminile in atto nel corso del XIX secolo. Pronta a difendere i suoi diritti e quelli delle altre donne, propone come soluzione la diffusione dell'istruzione.

La stessa Dora, sostenne che l'origine della disuguaglianza tra uomo e donna non era di natura biologica, ma di natura sociale. Quindi dipendeva da una tradizione culturale secondo cui le donne non dovessero istruirsi. Tutto questo aveva causato l'esclusione dalla politica delle donne e consentito l'emergere di false opinioni in merito alla loro inferiorità naturale. Un comportamento così ostile verso le donne Dora lo aveva notato in strutture tradizionali, il vero problema era liberare la società dagli antichi stereotipi riguardanti i ruoli femminili e quelli maschili.

Utilizzando fonti storiografiche, articoli e documenti d'archivio, la ricerca è volta a sottolineare l'importante ruolo che l'intellettuale rumena ebbe nel panorama europeo come scrittrice e donna impegnata in politica. L'obiettivo del lavoro è ricostruire il suo pensiero sulle donne attraverso le sue opere e i suoi contatti con gli intellettuali del tempo che apprezzarono in lei il pensiero sia politico che pedagogico.
\end{abstract}

Parole chiave: donna; istruzione; stereotipi; società; cultura femminile.

Abstract: Elena Ghika, known by the artistic name of Dora d'Istria, educated and erudite woman, is part of the debate on the female civil and cultural emancipation that took place during the nineteenth century. Ready to defend her rights and those of other women, she proposes as a solution the spread of education. 
Dora herself argued that the origin of inequality between men and women was not biological in nature but social in nature. So it depended on a cultural tradition that women did not have to educate themselves. All this had caused exclusion from women's politics and allowed the emergence of false opinions about their natural inferiority. Such hostile behavior towards women Dora had noted in traditional structures, the real problem was to free society from the ancient stereotypes about female and male roles.

Using historiographical sources, articles and archival documents, the research aims to underline the important role that the Romanian intellectual played in the European scene as a writer and a woman involved in politics. The aim of the work is to reconstruct his thoughts on women through his works and his contacts with intellectuals of the time who appreciated both political and pedagogical thought in her.

Keywords: woman; education; stereotypes; society; female culture.

SOMMARIO: 1. Introduzione. 2. La riflessione di Dora d'Istria sulla questione femminile. 3. Dora d'Istria in difesa del popolo Albanese. 4. Conclusioni. 5. Riferimenti bibliografici.

\section{INTRODUZIONE}

Nell'ampio dibattito che si stava aprendo in merito alla questione femminile nel coso del XIX secolo nell'area dell'Europa orientale, è d'obbligo un approfondimento della La principessa Elena Ghika, in arte Dora d'Istria, tra le donne più rinomate del XIX secolo. Scrittrice, scienziata, pubblicista, patriota e diplomatica, nonché sostenitrice della emancipazione femminile ${ }^{1}$.

Dora d'Istria, si inserisce nel panorama europeo con le sue idee innovative in merito all'educazione, sottolineando quanto fosse importante l'educazione morale del popolo attraverso l'educazione familiare. Usando lo pseudonimo Dora d'Istria² scrisse molti articoli in riviste europee, sottolineando in questo modo la sua cultura aperta, cosmopolita e tollerante.

La donna consacrò la sua vita all'attività intellettuale, scrisse molti testi, intrattenne molte relazioni epistolari con intellettuali di cultura arbëreshë (...) come Demetrio Camarda, uno dei primi attivisti del movimento culturale degli albanesi d'Italia, che le dedicò una raccolta di poesie dal titolo «A Dora d'Istria. Gli albanesi» ${ }^{3}$. Un ulteriore manifestazione di stima per la donna, per via della sua cultura e del

1 Elena Gjika Koltsova-Massalskaya, conosciuta con lo pseudonimo di Dora d'Istria (Bucarest, 22 gennaio 1828 - Firenze, 17 novembre 1888), romena di nascita, con origini albanesi, italiana per acquisizione, esponente del Romanticismo e del femminismo.

2 La ragione di questo pseudonimo era di natura nazionalista, infatti il suo nome d'arte sintetizzava il forte legame con la propria terra. Con il nome Dora D'Istria si riferiva a Dora dell'Istro, cioè il Danubio, il fiume che attraversa la Romania dopo aver percorso altre terre europee, dai monti tedeschi al Mar Nero. Un fiume che univa molti popoli diversi per storia, politica, religione e per la lingua.

${ }^{3}$ «A Dora D'Istria. Gli albanesi» è una raccolta di poesie di Demetrio Camarda, pubblicata a Livorno. 
suo spiccato senso critico, venne mostrato da Angelo de Gubernatis, un letterato, orientalista, professore di sancrito presso l'università di Firenze.

E' stata apprezzata da molti suoi contemporanei, ma anche da autori della prima metà del Novecento, credo proprio che tali apprezzamenti provenissero dalla sua straordinaria abilità da intellettuale consumata. Si legge che la sua perfetta conoscenza di varie lingue come: il russo, il greco, l'albanese, il romeno (sua lingua madre), la collocarono in posizioni privilegiate ${ }^{4}$. Anzi, pur essendo una donna, le sue abilità di scrittrice vennero comunque riconosciute da molti tra intellettuali e politici.

Ebbe l'opportunità di compiere molti viaggi, di osservare i comportamenti esteriori delle donne sia d'Europa che di altri continenti, confrontò le loro culture e tradizioni, nonché la condizione delle donne di fronte alla legge. Proprio durante questi suoi innumerevoli viaggi Dora D'Istria riuscì a scoprire l'universo femminile, presentando nelle sue opere i vari particolari femminili, dall'aspetto esteriore ai comportamenti.

\section{LA RIFLESSIONE DI DORA D'ISTRIA SULLA QUESTIONE FEMMINILE}

Elena Ghika, è stata una donna molto erudita, conosceva perfettamente il francese, l'inglese, il tedesco, il russo, il greco antico e quello moderno, l'albanese ed, ovviamente il rumeno, essendo la lingua della sua madrepatria. Usando lo pseudonimo di Dora d'Istria, scrisse parecchio, lasciandosi forse ispirare dai numerosi viaggi che ebbe la possibilità di compiere in Europa e non solo. Toccò argomenti di varia natura come la storia, la letteratura, la filosofia, al religione, le tradizioni popolari e i problemi sociali, ma ebbe sempre uno occhio particolare per le problematiche femminili ${ }^{5}$. Infatti, da esponente del Romanticismo e del Femminismo nell'Europa orientale, venne osservata e valutata con attenzione dai suoi contemporanei, nonché rispettata da molti intellettuali tra cui Paolo Mantegazza (Pommier, 1863; Yriarte, 1870; Cecchetti, 1868; Buloz, 1875). L'antropologo non solo la descrisse come "una delle menti più lucide e più intelligenti d'Europa», ma anche come "Un corpo tutto venustà, un cuor tutto grazia e nobiltà, una mente d'artista e di pensatore son tre cose rare a trovarsi, anche da sole, ma messe insieme formano un miracolo della fortuna; e questo miracolo ha saputo compiere la natura spargendo tutte quelle grandi e diverse virtù sopra un solo nome, quello di Elena Ghika, che diede poi a se stessa nel mondo della letteratura il secondo e più noto battesimo di Dora d'Istria» ${ }^{6}$.

${ }^{4}$ Per approfondimenti in merito alla cultura femminile si veda: Cagnolati, Covato (a cura di), 2016.

${ }^{5}$ Cfr. Rossi (a cura di), 1998.

${ }^{6}$ Cfr. Questa (s. d.). 
Scrisse sulle donne e per le donne, un esempio è rappresentato da "Les femmes en Orient», e tante altre opere ${ }^{7}$. Riuscì nell'intento di mettere a confronto culture e tradizioni differenti, perché a suo parere, fare riferimenti alla cultura di un paese significava valorizzarne le radici e le tradizioni.

Nell' opera Les femmes en Orient e Des femmes par une femme condusse determinati studi sulla condizione femminile in Europa orientale e occidentale. Una vera e propria storia delle donne ante litteram, nelle quali sono contenute un gran numero di informazioni sulle condizioni civili e sulle occupazioni delle donne del tempo.

Si partì dall'importanza dell'educazione, ai fini della loro missione di mogli, madri e istruttrici dei propri figli ai diritti civili e politici.

Un ulteriore compito che Dora d'Istria attribuì alle donne era la loro capacità di sensibilizzare in favore della pace. Da fervente sostenitrice degli ideali di libertà e di progresso dei popoli si schierò in favore della democrazia, in difesa dei più deboli ed oppressi. Un progetto strettamente collegato agli «ideali del Risorgimento che si tradusse nel riconoscimento dell'uguale dignità per tutti, in cui alle donne, educate per educare, si chiedeva l'impegno nel loro ruolo per contribuire a far diventare grande il proprio popolo» (Fidanzia, 2013, p. 94). A suo parere un modello da seguire era quello svizzero, l'unico paese d'Europa con un governo repubblicano.

In modo particolare in Italia, dopo le varie rivoluzioni curate dalle donne per ottenere maggiori diritti ad istruirsi, ci sono molti esempi di laureate, si veda Lidia Poet la prima donna laureata in Giurisprudenza a Firenze, Sibilla Aleramo scrittrice molto attenta ai temi femminili. La scrittura offriva sostanzialmente modelli in cui potersi immedesimare, un modello per le donne italiane (cfr. Soldani, 1991).

Da donna si espresse con forza anche per la difesa delle posizioni inerenti la condizione femminile nelle varie aree europee, diverse per cultura e tradizioni, dalle condizioni materiali, morali, sociali, politiche, giuridiche ed economiche. Così proprio nell'opera Les femmes en Orient, scrisse una disamina ragionata sulla condizione femminile ( $d$ 'Istria, 1859).

Analizzando il suo pensiero femminista si può notare come la donna riuscì non solo ad analizzare la condizione femminile a quell'epoca, ma pensò anche ad alcuni rimedi da adottare per rendere meno restrittiva la condizione della donna. Dimostrò l'importanza della educazione per le donne nel processo del progresso storico universale. Si può desumere che non è stato casuale l'interesse del giurista Carlo Francesco Gabba che proprio in quegli anni pubblicò «La questione femminile e la principessa Dora d'Istria» negli anni del dibattito sulla «riforma del Codice civile italiano» (Gabba, 1880), dei progetti per illuminare l'opinione parlamentare

\footnotetext{
7 Si consulti: d'Istria, 1859; 1865; 1871; 1872; Osman-Bey, 1883.
} 
ed extraparlamentare sui bisogni delle donne, sulle ingiuste esclusioni, quando la donna era una parentesi nel Codice civile ${ }^{8}$.

L'intellettuale inventò la parola emancipazione, una parola per cui si è molto abusato e per la quale spesso sono stati diminuiti l'importanza ed il rispetto dovuti alla missione della donna. L'obiettivo principale di una donna è proprio quello di ridare vita e colore ad immagini sfocate dal passato, di riportare nitore alle tinte sbiadite.

Ella, per esempio, testimoniò su alcuni aspetti di vita delle donne albanesi per quanto riguardava la loro appartenenza alla vita politica del loro paese e del loro impegno in questioni di pace nei casi in cui era necessaria una conciliazione e definì gli albanesi come «i migliori soldati in oriente». Allo stesso livello dei vicini serbi, divisi tra tre confessioni religiose, cattolica, ortodossa musulmana (d'Istria, 1865, p. 268).

L'autrice continuò le sue osservazioni sul Paese delle aquile, dividendolo in quattro regioni, ognuna con caratteristiche socio-culturali distinte e nelle quali la condizione della donna cambiava in modo considerevole. Per esempio, in Mirdita, i monaci cattolici ispirarono nelle donne un fervente spirito anti-scismatico. Nella regione settentrionale, «dall'età di sedici anni le donne mirdite marciavano con delle pistole alle cinture, scortate da certi mastini che gli antichi chiamavano molossi. Svelte e fiere, estranee a ogni piacere» (d'Istria, 1865, p. 268). Invece, per descrivere le caratteristiche delle donne Tosk, nel Sud del Paese e per parlare del "loro carattere e della loro influenza», narrava le gesta della famiglia di Alì Paschà, che avrebbe «inondato la sua patria di torrenti di sangue e al tempo stesso di prodigi di eroismo». "Tutte le considerazioni che potremmo fare sulle donne musulmane dell'Albania sarebbero insignificanti comparate alla vita di Khamco e di sua figlia Käinitza», madre e sorella del regnante (Ivi, 1865, pp. 271-272).

II racconto continuava in questo modo: «Ed ecco che Khamko, morto il marito, riesce a organizzare e raccogliere attorno a sé un piccolo esercito per combattere le popolazioni confinanti. Sconfitta e imprigionata insieme alla figlia nella prigione di Gardiki, coverà la vendetta e instillerà nel cuore del figlio lo stesso sentimento, in seguito accompagnato nelle velleità di conquista dalla sorella Käinitza (o Chäinitza), che nel frattempo per seguirlo aveva abbandonato il marito». Le gesta delle donne albanesi in battaglia, forti e valorose, determinate e combattive, percorrono tutta l'opera di Dora D'Istria, da Nord a Sud, dalla Mirdita alla Djamouria, dalla Toska alla Liapouria «Sobrio, sveglio, intelligente, senza piacere per lo studio e considerazione per l'agricoltura, l'Albanese è prima di tutto soldato» (Ivi, 1865, p. 328).

La sua descrizione è concentrata sulla condizione delle donne, dal punto di vista delle tradizioni e della cultura delle due metà del continente europeo, compresa la Romania, la Bulgaria e l'Albania. Per questo suo interessamento ricevette l'approvazione anche dal generale Giuseppe Garibaldi il quale la chiamò «un eroe, sorella,

\footnotetext{
${ }^{8}$ Cfr, Borcia, 2014.
} 
un'anima volta ai più alti ideali» (Mondolfo, 1959). L'eroe dei due mondi, era convinto che le donne avessero un ruolo importante nel processo di emancipazione delle nazionalità oppresse e nella lotta al dispotismo e alla superstizione.

Analoghe considerazioni sulla necessità dell'educazione femminile vennero avanzate da una serie di intellettuali e politici italiani nello stesso periodo, uno fra i tanti è stato Salvatore Morelli con la sua numerosa azione letteraria e politica in favore delle donne, appoggiato da Giuseppe Mazzini e da Stuart Mill ${ }^{9}$.

Poi ci furono le azioni condotte dalle contadine russe che erano tenute in uno stato di completa ignoranza (D’Alessandri, 2007, p. 223). Dora difese tutte le donne, poiché era convinta che avrebbero dovuto coordinarsi fra loro per oppose resistenza ad ogni tipo di guerra, impegnandosi in modo concreto per la pace. Toccava alle madri, quindi alle donne, far sviluppare nei propri figli un profondo spirito di fratellanza fra tutti gli uomini.

Sostanzialmente, Dora d'Istria è stata una valida mediatrice fra gli italo -albanesi, i greci e gli italiani in vista di una soluzione complessiva della questione d'Oriente e di quella italiana, tale funzione di mediazione venne svolta nel corso dell'Ottocento proprio dagli intellettuali citati, nonché dai viaggiatori.

Ha "precorso di un secolo le idee sull'educazione femminile», come ha ben sottolineato Roberta Fidanzia, idee che si sono poste in una via alternativa sia al discorso della emancipazione intesa in modo sbagliato, sia sull'obbligata ignoranza (Fidanzia, 2013, p. 95).

Queste idee conducono «alla vera schiavitù della donna (...) Fino ad ora, purtroppo, non solo da noi, ma come risulta dalle osservazioni della nostra scrittrice, si è coltivata più la debolezza che la forza della donna, sempre soggetta, sempre schiava. (...) Oggi, finalmente, si è compreso che per la donna è necessaria l'istruzione per se tessa, per l'educazione dei figli propri e per quelli degli altri, che possono venire affidai alle sue cure e al suo appoggio morale e intellettivo. (...) L'amore nella donna è più profondo che nell'uomo, ed assume quella parte importante che anima la donna stessa e che importa tutte le opere della sua vita» (Santi, 1917, pp. 22-23).

\section{DORA D'ISTRIA IN DIFESA DEL POPOLO ALBANESE}

Dora d'Istria è conosciuta per la sua intraprendenza, cultura e per lo sguardo attento ed aperto, tipico di chi è abituato a viaggiare, guardandosi intorno, cogliendo i vari aspetti delle culture che incontra. Se per un verso rimase affascinata dalle cose più belle che vide, per esempio in Italia, in Liguria, per altri aspetti si innamorò anche dei paesaggi rurali, perché come si è già detto ella osservava le tradizioni le questioni sociali, facendo riferimento ai popoli ed alle donne, al loro ruolo nella

\footnotetext{
${ }^{9}$ Su questo argomento si consulti: Bosna, 2013.
} 
società, alla loro libertà. Si soffermò a studiare le donne albanesi, il loro ingegno, ma anche le loro difficoltà di libera espressione.

In merito a questo problema sociale si scontarono riformisti propensi ad un riscatto sia morale che civile delle donne ai fini di poter operare una modernizzazione del Paese, mentre erano di parere opposto i conservatori, secondo i quali la donna doveva rimanere subordinata all'uomo. L'Albania si mostrò divisa in due parti anche da questo punto di vista. Così nel libro Les femmes en Orient, si parlò di un possibile miglioramento attraverso l'introduzione dell'istruzione femminile di cui, probabilmente, la d'Istria si rese unica rappresentante nel contesto del dibattito emancipazionista dell'epoca.

D'Alessandri scrive che «quella albanese è la terza nazionalità balcanica alla quale Dora d'Istria dedicò maggiore attenzione» (D’Alessandri, 2007, p. 181). La nobile donna infatti, da coraggiosa sostenitrice della causa albanese per via della sua discendenza paterna, volle sensibilizzare le altre popolazioni a tale causa, visto il momento di particolare difficoltà per una nazione ancora sotto l'occupazione dell'Impero ottomano (Ibidem, p. 181). In quella situazione gli intellettuali di origine europea e gli arbëreshe, ovvero gli italo - albanesi, ebbero un ruolo importante.

Non è un caso che Demetrio Camarda ${ }^{10}$, uno dei primi attivisti del movimento culturale degli albanesi d'Italia, nel 1870 presentasse una raccolta di poesie dal titolo «A Dora d'Istria. Gli albanesi» (Greco, 1875, pp. 73-74). Camarda, è rinomato anche per il suo impegno di linguista di cui diede ampia dimostrazione nel tempo e di cui non si può non citare il saggio dal titolo l'Alfabeto generale epirotico (1869), nel quale lanciò l'idea di un alfabeto comune, in aggiunta ad uno studio sulla principessa dal titolo "A Dora d'Istria». In parallelo aveva anche molta stima anche per Geronimo De Rada ${ }^{11}$, altro intellettuale arbëreshe ${ }^{12}$, con cui la donna era in contatto. Ella scrisse: «Egregio e caro signor Geronimo, la mia onorata Patria è l'Arbëria, e ad essa apparterrò fino alla fine della mia vita...» (d'Istria, 1859-1860).

${ }^{10}$ Demetrio Camarda, nato a Piana degli albanesi, linguista italiano di origine arbëreshe, è stato un importante filologo, fra i primi studiosi della linguistica albanese. Scrisse nel 1864 un saggio di grammatologia comparata sulla lingua albanese, poi scrisse un'Appendice al Saggio, nel 1866. E' rinomato per la sua raccolta di canti popolari italo-albanesi. Sprovë e gramatologjisë krahasuese mbi gjuhën shqipe è la prima opera scientifica di studio storico-comparativo sull'argomento.

${ }^{11}$ Geronimo De Rada, nato a San Demetrio Corone in provincia di Cosenza, ha dato lustro alla storia degli Albanesi, una minoranza linguistica storica tra le più consistenti in Italia. Per la sua molteplice attività culturale, è ritenuto «il Vate» di tutti gli Albanesi d'Albania, scrisse fra le altre nel 1886 l'opera i Canti di Milosao (figlio del despota di Scutari). E' considerato il padre della letteratura arbëreshe, oltre che l'iniziatore della letteratura albanese moderna.

12 Gli italo-albanesi che ebbero un ruolo fondamentale nel processo di rinascita nazionale albanese durante la Rilindja. 
Questa sua attenzione ed attaccamento alla causa albanese è documentata da una fitta corrispondenza con De Rada con il quale costituì una "Associazione letteraria» ed un'«Accademia albanese». Per di più, comunicò anche l'intenzione di comporre una grammatica albanese presso la biblioteca nazionale di Firenze, perché, l'inizio del cambiamento del popolo albanese doveva compiersi attraverso l'educazione. La realizzazione di scuole in cui si parlasse la lingua nazionale, seguendo un programma scolastico che il De Rada curò con molto impegno (D'Alessandri, 2006).

$\mathrm{Si}$ intraprese finanche la strada della scrittura giornalistica, strumento ideale per la diffusione delle notizie. Alcuni dei giornali più interessanti sono: Shqypeja e Shqipnise (L'albanese d'Albania), Fiàmuri Arberit (La bandiera dell'Albania) e del quotidiano Shpresa Kombëtare (La speranza nazionale) di Marigo Pozio il cui primo numero apparve a Vlora nel 1921. Compose alcuni saggi su questo popolo in cui parlò del popolo albanese e della sua eredità culturale e artistica, della ricca letteratura orale, del folclore, dei rari ed unici vestiti tradizionali di tutte le regioni albanesi. Si ricordano: «Kombësia Shqiptare, sipas këngëve popullore» e «Shqiptarët e të dyja anëve të Adriatikut» (La Nazionalità Albanese, secondo i canti popolari» e "Gli Albanesi di entrambe le sponde dell'Adriatico) (D'Alessandi, 2006).

Quindi, era chiaro che l'Arbëria, come precisò Dora d'Istria in una lettera indirizzata al signor De Rada, «era e sarebbe sempre stata la sua Patria onorata alla quale si sarebbe sentita legata fino alla fine della sua vita» (D'Alessandri, 2007).

\section{CONCLUSIONI}

L'impegno di Dora D'Istria è stato senza dubbio molto rilevante nella società del XIX secolo. II suo approccio alla realtà, non solo quella delle donne, ma anche della società in genere è connotata da una indiscussa capacità di apertura verso l'altro, nei suoi elementi più concreti e nell'atto di cogliere in modo intuitivo gli aspetti particolari della realtà mettendoli in relazione con la totalità dell'essere.

Si soffermò su vari aspetti della vita tra cui quello dell'istruzione femminile, tema principale in questo lavoro. Del resto, come afferma Sami Frashëri

"Dare istruzione ad un uomo significa piantare un albero che fa solo ombra, mentre istruire una donna significa piantare un albero che oltre a fare ombra dà anche frutti».

Nella storia del genere umano ci sono state poche donne che si sono distinte in vari campi, e quelle che ci sono riuscite è stato per via dell'appoggio dato loro da parte di uomini famosi.

Molte donne sono rimaste nell'oscurità e sono state indicate soltanto con il nome «donna».

Dora d'Istria ha fornito indubbiamente un valido contributo al confronto di idee sulla questione femminile alimentatosi in tutta l'Europa occidentale. Mostrò 
attenzione e sensibilità per la causa femminile, prestando attenzione alle differenze reali esistenti tra i diversi popoli. In questa fase della storia le donne albanesi, tra alti e i bassi, si sono distinte nelle loro attività ed impegni sociali. La scrittrice, ha passato in rassegna questi cambiamenti che rientrarono nel fenomeno di rinascita del Risorgimento albanese alimentato anche dall'impegno di donne dello stampo delle sorelle Sevasti e Parashqevi Qirjazi, nello sforzo di istruire la popolazione femminile attraverso la «Scuola delle ragazze» (Dishnica, 1997).

\section{RIFERIMENTI BIBLIOGRAFICI}

Ales Ballo A. e Brezzi F. (a cura di). (2001). Il filo(sofare) di Arianna. Percorsi del pensiero femminile nel Novecento. Milano: Mimesis.

Bosna V. (2013). Salvatore Morelli in difesa delle donne nell'Italia risorgimentale. Roma: Aracne.

Borcia, O. D. (2014). Dora d'Istria, messaggera della pace e dell'unione tra Oriente e occidente. Analele Universitatii Crestine Dimitrie Cantemir, Seria Stiintele Limbii, Literaturii si Didactica predarii, 2014-2, pp. 83-95. <http://aflls.ucdc. ro/II_2014/Otilia\%20Doroteea\%20Borcia\%20\%20Dora\%20d\%20lstria\%20 messagera\%20della\%20pace\%20e\%20dell\%20unione\%20tra\%20oriente\%20 ed\%20occidente.pdf>

Buloz, F. (1875). Revue des deux mondes. Paris.

Cagnolati A. e Covato C. (a cura di). (2016). La scoperta del genere tra autobiografie e stili di vita. Siviglia: Benilde.

Cecchetti, B. (1868). Bibliografia della Principessa Elena Ghika, Dora d'Istria. Venezia.

Cecchettii B. (1808). Di alcune opere della principessa Dora D'lstria. Venezia: Tipografia del commercio di Marco Visentini.

D'Alessandro A. (2007). Il pensiero e l'opera di D'Ora d'Istria fra oriente europeo e Italia. Roma: Istituto per la Storia dl Risorgimento italiano, Gangemi ed.

D'Istria, D. (1856). La Suisse allemande e I'ascension du Moench. Vol. III. ParisGenéve: Cherbuliez. 
D'Istria, D. (1959). Les femmes en Orient. La Péninsule Orientale, 1 Vol. Zurich: Merier \& Zeller.

D'Istria, D. (1859-1860). Les femmes en Orient. Zurich: Meyer \& Zeller (2 Voll.). Vol. 1. Cherbuliez, 1863.

D'Istria, D. (1863). Excursions en Roumélieet en Morée. Zurich: Meyer et ZellerParis: J. Cherbuliez.

D'Istria, D. (1865). Des par une femme. Paris, Lacroix: Verboeckhoven.

D'Istria, D. (1871) Les femmes fortes, comedie in trois actes ed prose, pare Victorien Sardu, Michel e Levy Freres Ed., a la Librerie Nouvelle. Paris.

D'Istria, D. (1872). Lettre à la presidente de l'Association des dames grècques pour I'istruction des femmes.

D'Istria, D. (1873). Gli albanesi in Rumenia, Storia dei principi Ghika, nei secoli XVII, XVIII e XIX su documenti inediti degli archivi di Venezia, Vienna, Parigi, Berlino, Costantinopoli ecc., estratto da «Rivista europea». Firenze: Tipografia editrice dell'associazione.

Dishnica, D. (1997). Motrat Qiriazi. Tiranë: Enciklopedike.

Fidanzia R. (2013). Dora D'Istria uno sguardo femminile sull'Ottocento. Risorgimento, Pedagogia, Politica, Condizone femminile. Roma: Aracne, Roma.

Gabba C. F. (1880). Della condizione giuridica delle donne. 2. a ed. Torino.

Greco O. (1875). Bibliografia femminile italiana del XIX secolo. Venezia: Mondovì, Tip. Gio. Issoglio.

Mondolfo R. (1959). Il pensiero politico nel Risorgimento italiano. Milano: Nuova Accademia Editrice, Milano.

Osman-Bey (Major Vladimir Andrejevich). (1883). Les femmes en Turquie par Osman-Bey Major Vladimir Andrejevich. Paris: Nouvell Ed.

Pommier, A. (1863). Madame la comtesse Dora d'Istria. Brussels. 
Questa M. (s. d.). Elena Ghika (Dora D'Istria) - Storia di una principessa romena a Firenze, negli anni immediatamente precedenti la nascita di Dino Campana. $<w w w . c a m p a n a d i n o . i t / i n d e x . p h p ? .$. dora-distria> (consultata il 24/06/2019).

Rossi, L. (a cura di). (1998). D. d'Istria, I bagni di mare. Una principessa europea alla scoperta della Riviera. Genova: Sagep.

Santi I. (1917). Dora D'Istria e le sue idee intorno all'educazione della donna. Estratto della «Rivista Pedagogica» diretta da Luigi Credaro, Anno X-Fasc, 5-6. MilanoRoma-Napoli: Società Editrice Dante Aligheri.

Soldani, S. (a cura di). (1991). L'educazione delle donne. Scuole, modelli di vita femminile nell'Italia dell'Ottocento. Milano: F. Angeli.

Yriarte, Ch. (1870). Portraits cosmopolites. Paris, 1870. 